\title{
Review of: "Effects of infectious disease consultation and antimicrobial stewardship program at a Japanese cancer center: An interrupted time-series analysis"
}

Tohru Takata ${ }^{1}$

1 Fukuoka University

Potential competing interests: The author(s) declared that no potential competing interests exist.

IIn this paper, the authors investigated the effectiveness of ID consultations and ASP at a Japanese cancer center for 36 months by interrupted time series analysis comparing Phase 1 and Phase 2. The main findings were 1) In Phase 2, monthly carbapenem days of therapy decreased significantly, and narrowspectrum antibiotic usage increased significantly in both trend and level. 2) Monthly DOT of antipseudomonal agents decreased significantly in trend and level. 3) The trend of multidrug-resistant $P$. aeruginosa and MRSA infections decreased without changes in the incidence of resistant organism, allcause in-hospital mortality, and length of stay, while the total number of inpatient specimens were significantly increased. Thus they conclude combining ID consultation and ASP reduced carbapenem use without negative patient outcomes.

<General Comments>

The design of the study is solid and the manuscript is well written. Although most of the findings are within the scope of similar previous reports on ID consultation and ASP, the value of this study lies in the subject specialized to cancer center.

$<$ Specific Comments $>$

1. It is of interest for the change of use of oral fluoroquinolones with antipseudomonal effect such as levofloxacin and ciprofloxacin between the two phases since it seems to be commonly prescribed for oral switch in cancer patients with neutropenia.

2. (Abstract, last sentence) 'improve prognosis in cancer patients' $\rightarrow$ Direct evidence supporting this conclusion was not shown in the text. 